\title{
Globular clusters : Ideal laboratories to test hydrogen-burning nucleosynthesis and hydrodynamics in stars?
}

\author{
Corinne Charbonnel \\ Geneva Observatory - 51, chemin des Maillettes - 1290 Sauverny - Switzerland \\ CNRS UMR 5572 - LATT - 14, av.E.Belin - 31400 Toulouse - France \\ E-mail: Corinne.Charbonnel@obs.unige.ch
}

\begin{abstract}
Galactic globular cluster (GC) stars exhibit abundance patterns which are not shared by their field counterparts, namely the well-documented $\mathrm{C}-\mathrm{N}, \mathrm{O}-\mathrm{Na}$ and $\mathrm{Mg}-\mathrm{Al}$ anticorrelations. Recent observations provided compelling evidence that these abundance anomalies were already present in the intracluster gas from which the presently observed stars formed. A widely held hypothesis is that the gas was polluted early in the history of the GC by material processed through $\mathrm{H}$ burning at high temperature and then lost by stars more massive than the observed long-lived stars. However the "polluters" have not been unambiguously identified yet. Most studies have focused on AGB stars, but rotating massive stars present an interesting alternative. In this talk we critically analyse the pros and cons of both potential stellar polluters. We discuss the constraints that the observational data bring on stellar nucleosynthesis and hydrodynamics as well as on nuclear reaction rates and we try to answer to the following question : "Are GC ideal laboratories to test stellar nucleosynthesis and hydrodynamics?"
\end{abstract}

International Symposium on Nuclear Astrophysics - Nuclei in the Cosmos - IX

June 25-30 2006

CERN, Geneva, Switzerland 


\section{Chemical properties of the galactic globular clusters. Evidences for early pollution of the intra-cluster material}

Globular clusters (hereafter GC) are extremely important astrophysical objects. These large aggregates of coeval stars can be used as cosmological tools, they bring clues on the formation and early evolution of galaxies as well as on the interactions and merging processes between galaxies. Last but not least, they are excellent laboratories to test the theory of stellar evolution. However, and despite their broad astrophysical importance, large uncertainties remain regarding their origin and formation processes as well as their early dynamical and chemical evolution. For all these reasons GCs have been extensively studied since the early fifties.

During the last three decades, an incredible amount of data has been collected in order to depict the chemical properties of galactic GCs thanks to high spectral resolution abundance analysis. We refer to Gratton et al. (2004) and to Sneden (2005) for recent and extended reviews on the observed abundance trends in GCs. We briefly recall here the main points : (i) Individual GCs appear to be fairly homogeneous as far as the iron peak elements $(\mathrm{Ni}, \mathrm{Cu})$ are concerned (with the notable exception of $\Omega$ Cen); (ii) They present very low scatter and the same trends as field stars for the neutron-capture elements (both s- and r-; $\mathrm{Ba}, \mathrm{La}, \mathrm{Eu}$ ) and the alpha-elements ( $\mathrm{Si}, \mathrm{Ca}$ ); (iii) They exhibit however specific patterns and large star-to-star abundance variations for the lighter elements from $\mathrm{C}$ to $\mathrm{Al}$ which are not shared by their field counterparts. Let us focus on these light element "anomalies".

After the pioneer work of Osborn (1971), several studies showed that GCs are very heterogeneous in $\mathrm{C}$ and $\mathrm{N}$, and that anticorrelated variations in molecular band-strengths of $\mathrm{CN}$ and $\mathrm{CH}$ exist all the way down to the main sequence turnoff in all the GCs where data are available. Starto-star variations of the strengths of the lines of $\mathrm{Na}$ and $\mathrm{Al}$ at the tip of the RGB were discovered at the end of the seventies (Cohen 1978; Peterson 1980; Norris et al. 1981). An anti-correlation between O and Na abundances (Pilachowski 1989, Drake et al. 1992 and Sneden et al. 1991 for the earliest studies) exists among evolved RGB stars in all the GCs studied to date. Whenever all the necessary data are available, $\mathrm{N}$ is found to be anticorrelated with $\mathrm{O}$, which itself positively correlates with $\mathrm{C}$, and the sum $\mathrm{C}+\mathrm{N}+\mathrm{O}$ appears to be constant within the observational errors (e.g., Dickens et al. 1991, Ivans et al. 1999). In addition, variations in $\mathrm{Al}$ and $\mathrm{Mg}$, again correlated with each other, have been discovered (e.g. Shetrone 1996, Ivans et al. 1999, Ramirez \& Cohen 2002). The sum $\mathrm{Mg}+\mathrm{Al}$ is also found to be constant in several clusters (Shetrone 1006). Last but not least, observations in NGC 6752, M 13 and M 71 show that there exist a large spread in the ratio ${ }^{24} \mathrm{Mg}:{ }^{25} \mathrm{Mg}:{ }^{26} \mathrm{Mg}$ together with a positive correlation between ${ }^{26} \mathrm{Mg}$ and $\mathrm{Al}$ as well as an anticorrelation between ${ }^{24} \mathrm{Mg}$ and $\mathrm{Al}$ but no correlation between ${ }^{25} \mathrm{Mg}$ and $\mathrm{Al}$ (Yong et al. 2003, 2005, 2006). All these features are considered anomalous because they are not seen in field stars of similar metallicity (e.g., Gratton et al. 2000).

It was soon recognized that the observed abundance patterns are the signatures of the simultaneous operation of the $\mathrm{CNO}, \mathrm{NeNa}$ and $\mathrm{MgAl}$ cycles of hydrogen-burning (see Fig. 1; Denissenkov \& Denissenkova 1990, Langer et al. 1993; Langer \& Hoffman 1995). Proton-captures on ${ }^{16} \mathrm{O}$ and ${ }^{22} \mathrm{Ne}$ respectively lead to the destruction of ${ }^{16} \mathrm{O}$ and to the production of ${ }^{23} \mathrm{Na}$ at temperatures $>2 \times 10^{7} \mathrm{~K}$. At higher temperatures $\left(\sim 4 \times 10^{7} \mathrm{~K}\right),{ }^{20} \mathrm{Ne}$ generates ${ }^{23} \mathrm{Na}$. On the other hand the $\mathrm{MgAl}$-chain is only active at temperatures higher than $\sim 7 \times 10^{7} \mathrm{~K}$ (Arnould et al. 1999; Prantzos 


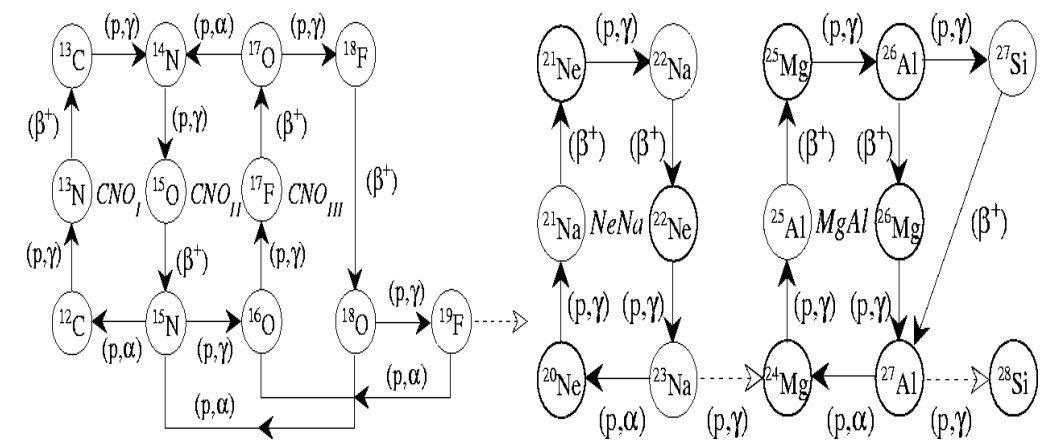

Figure 1: Reactions of the CNO-cycles and NeNa- and MgAl-chains. The dashed lines present the possible leakages out of the cycles. Taken from Arnould et al. (1999)

\& Charbonnel 2006b, hereafter PC06b).

Although the underlying nucleosynthesis is unambiguous, the identification of the corresponding astrophysical site still remains a challenge. For a long time the main question was to know if such patterns were inherited at the birth of the stars that we are currently observing (the so-called self-enrichment or primordial hypothesis which implies pollution of the intracluster gas by more massive and faster evolving stars) or if they were generated in the course of the evolution of these objects (the so-called evolution hypothesis which requires non-canonical mixing inside the star itself). A major breakthrough was made in that respect from high resolution spectrographs on very large telescopes that allowed one to determine the abundances of the concerned elements in faint subgiant and turnoff stars in a couple of GCs (Gratton et al. 2001; Thévenin et al. 2001; Grundhal et al. 2002; Carretta et al. 2003, 2004; Ramirez \& Cohen 2002, 2003). The discovery of the $\mathrm{O}-\mathrm{Na}$ and $\mathrm{Mg}-\mathrm{Al}$ anticorrelations in these scarcely evolved objects has given a new spin to the self-enrichment scenario. Indeed turnoff stars are not hot enough for the required nuclear reactions to occur in their interiors. This implies that the $\mathrm{C}, \mathrm{N}, \mathrm{O}, \mathrm{Na}, \mathrm{Mg}$ and $\mathrm{Al}$ abundance anomalies pre-existed in the material out of which these objects formed; their gas must have been polluted early in the history of the cluster by more massive and faster evolving stars (Cottrell \& Da Costa 1981).

To summarize, the emerging scenario is that the GC stars that we observe now must have formed from the mass processed through proton-capture nucleosynthesis via the CNO-cycle and the NeNa- and MgAl-chains and then lost (perhaps mixed with some original material) by a first generation of more massive and faster evolving objects. This first generation consists of the original cluster population and shares the chemical composition of the field stars with similar metallicity. But what type of stars did shape the abundance anomalies?

Because we have very strong nucleosynthesis constraints, coming mainly from the observed $\mathrm{Mg}$ isotopic ratio, we can get clues on the potential polluters by looking at the temperature at which hydrogen burning occurs in various types of stars. Fig. 2 shows the maximum temperature reached in the H-burning regions for stars of various initial masses at different stages of their evolution. The horizontal black lines delimit the temperature range of $\mathrm{H}$-burning within the progenitors of the chemical anomalies, as constrained by the combination of the observational data for $\mathrm{Na}, \mathrm{Al}$ and the $\mathrm{Mg}$ isotopes (see PC06b). One sees that there are actually two potential polluters : massive AGB 


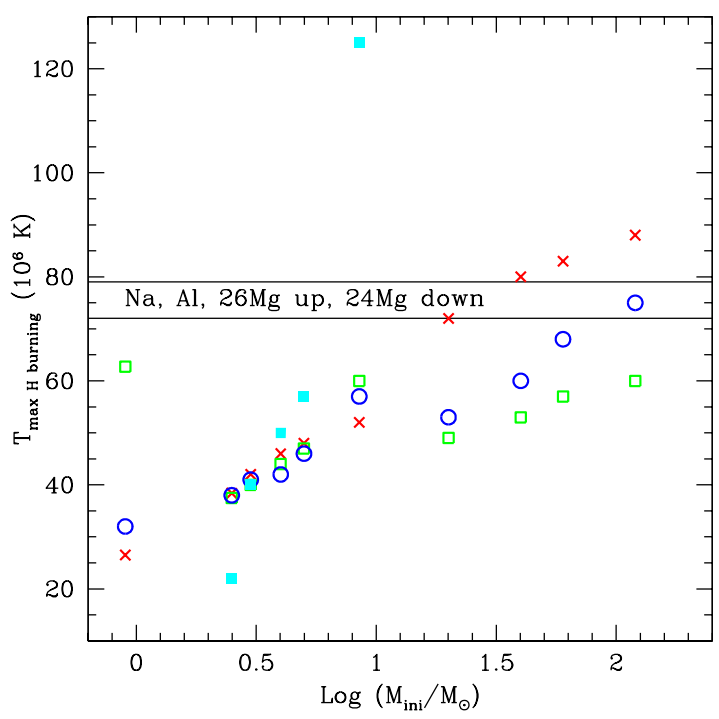

Figure 2: Maximum temperature reached in the H-burning regions for stars of various initial masses (and with $[\mathrm{Fe} / \mathrm{H}]=-1.5)$ at different phases of their evolution : In the core at the end of the main sequence (red crosses); in the hydrogen-burning shell at the red giant tip (i.e., before central He-ignition; green open squares) and on the clump (i.e., during central He-burning; blue circles); at the base of the convective envelope on the TP-AGB (cyan filled squares). The horizontal black lines delimit the temperature range where H-burning occurs in the progenitors of the abundance anomalies, as constrained by the combination of the observed abundances of $\mathrm{Na}, \mathrm{Al}$ and of the $\mathrm{Mg}$ isotopes (see Prantzos \& Charbonnel 2006b for more details).

stars, and massive stars.

\section{Were AGB stars the polluting agents?}

Usually it is claimed that massive Asymptotic Giant Branch stars (AGBs) are responsible for the observed composition anomalies in GCs (Cottrell \& Da Costa 1981). These objects indeed present several qualitative advantages that make them very attractive within the primordial scenario: (1) They possibly experience the so-called hot bottom burning (hereafter HBB) which processes their large envelopes through the required $\mathrm{CNO}, \mathrm{NeNa}$ and $\mathrm{MgAl}$ nucleosynthesis ${ }^{1}$; (2) They do not produce $\alpha$-nor Fe-peak elements; (3) Their low-speed winds may be retained within the cluster; (4) The UV energy produced during the planetary nebulae phase is too low to expel the gas away; (5) Their lifetime (50 - $100 \mathrm{Myr}$ ) is low enough to be compatible with the GC formation.

Figures 3 and 4 summarize qualitatively the nucleosynthetic paths and mixing events which occur in TP-AGB stars and which are relevant for the $\mathrm{O}-\mathrm{Na}$ and $\mathrm{Mg}-\mathrm{Al}$ abundance problems (see

\footnotetext{
${ }^{1}$ In their pioneer paper Cottrell \& Da Costa (1981) suggest that $\mathrm{Na}$ and $\mathrm{Al}$ enrichment in CN-strong stars of the GCs 47Tuc and NGC 6752 might be produced within intermediate mass stars $\left(\sim 5-10 \mathrm{M}_{\odot}\right)$. However the invoked process is neutron-captures on ${ }^{22} \mathrm{Ne}$ and ${ }^{25} \mathrm{Mg}$ within the thermal pulse (the neutrons being released by the ${ }^{22} \mathrm{Ne}(\alpha, \mathrm{n}){ }^{25} \mathrm{Mg}$ reaction; see Iben 1976).
} 


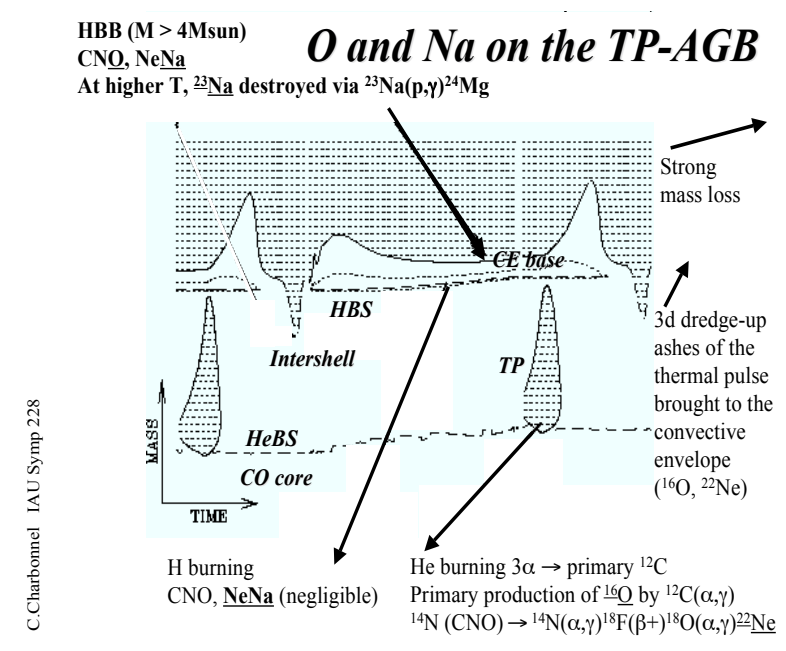

Figure 3: Kippenhahn diagram representing schematically the evolution of the internal structure of an AGB star between two successive thermal pulses (The shaded parts represent the convective regions.). The nucleosynthetic paths and mixing events relevant for the building up of the $\mathrm{O}-\mathrm{Na}$ anticorrelation are indicated (Adapted from Mowlavi 1998).

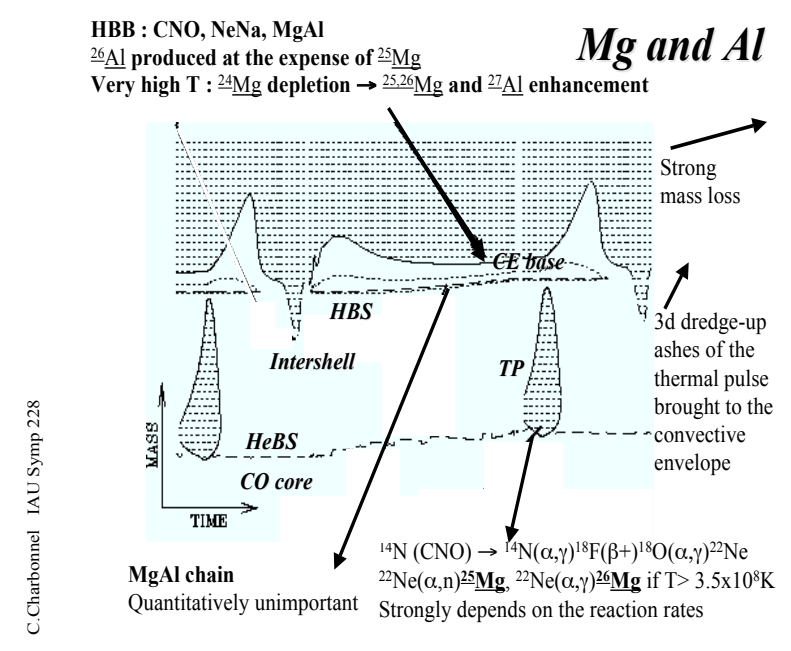

Figure 4: Same as Fig.3 but for the $\mathrm{Mg}-\mathrm{Al}$ anticorrelation

Mowlavi \& Meynet 2000 and Karakas \& Lattanzio 2003 for more details on the production of the $\mathrm{Mg}$ and $\mathrm{Al}$ isotopes).

The competition between the $3 \mathrm{~d}$ dredge-up events and the HBB is crucial for the final AGB yields. On one hand indeed, the $3 \mathrm{~d}$ dredge-up is expected to bring to the stellar surface primary ${ }^{16} \mathrm{O}$ (in uncertain quantities), as well as (primary) ${ }^{22} \mathrm{Ne}$ and ${ }^{25,26} \mathrm{Mg}$ produced via successive $\alpha$-captures on ${ }^{14} \mathrm{~N}$ in the convective tongue during the thermal pulses. This production of the neutron heavy $\mathrm{Mg}$ isotopes in the He-shell flash via ${ }^{22} \mathrm{Ne}(\alpha, \mathrm{n}){ }^{25} \mathrm{Mg}$ and ${ }^{22} \mathrm{Ne}(\alpha, \gamma){ }^{26} \mathrm{Mg}$ depends on the abundance of the matter left by the $\mathrm{H}$-shell at the end of the interpulse phase. It requires temperatures higher than $\sim 3 \times 10^{8} \mathrm{~K}$ which are reached typically in the He-shell for stars initially more massive 
than $\sim 3 \mathrm{M}_{\odot}$ and strongly depends on the reaction rates. The $\mathrm{Al}$ isotopes are not produced in the He-shell.

On the other hand, if the bottom envelope temperature is high enough (typically for stars with masses higher than $\sim 4 \mathrm{M}_{\odot}$ ), HBB further modifies the envelope abundances via the CNO-cycle and the $\mathrm{NeNa}$ and $\mathrm{MgAl}$ chains. HBB results in the production of ${ }^{14} \mathrm{~N}$, in the depletion of ${ }^{15} \mathrm{~N}$, ${ }^{18} \mathrm{O}$, and, if the temperature is high enough, of ${ }^{16} \mathrm{O}$. When the NeNa- and $\mathrm{MgAl}$-chains operate, ${ }^{23} \mathrm{Na}$ and ${ }^{26} \mathrm{Al}$ are produced at the expense of the dredged-up ${ }^{22} \mathrm{Ne}$ and ${ }^{25} \mathrm{Mg}$ respectively (Note that an important increase of the surface ${ }^{23} \mathrm{Na}$ abundance already occurs as a result of the second dredge-up from the conversion of the initial abundance of ${ }^{22} \mathrm{Ne}$ and some ${ }^{20} \mathrm{Ne}$ by $\mathrm{H}$-shell burning). In the case of very high $\mathrm{HBB}$ temperatures, proton-captures on ${ }^{23} \mathrm{Na}$ and ${ }^{24} \mathrm{Mg}$ deplete the surface abundances of the elements, and some ${ }^{25,26} \mathrm{Mg}$ and ${ }^{27} \mathrm{Al}$ are produced.

Several custom-made detailed models were recently computed in order to test the AGB hypothesis (Ventura et al. 2001, 2002; Denissenkov \& Herwig 2003; Karakas \& Lattanzio 2003; Herwig 2004a,b; Ventura \& D'Antona 2005a,b,c). Most of these studies concluded that the AGB pollution scenario suffers from severe drawbacks from the point of view of the nucleosynthesis (e.g., Fenner et al. 2004, Charbonnel 2005). These difficulties stem from the subtle competition between HBB and third dredge-up (which contaminates the stellar envelope with the products of helium burning). The problems can be summarized as follows : In TP-AGB stars, (i) O is not depleted to the extent required by the observations, while $\mathrm{Na}$ is over-produced or over-destroyed depending on the treatment of convection; (ii) $\mathrm{Mg}$ is produced while it should be destroyed, and the $\mathrm{Mg}$ isotopic ratio is in conflict with the (rare) available data; (iii) $\mathrm{C}+\mathrm{N}+\mathrm{O}$ does not remain constant in AGB processed material, in contrast to observations (Dickens et al. et al. 1991; Smith et al. 1996; Smith et al. 2005; Ivans et al. 1999). Rotation-induced mixing makes the situation even worse (Decressin \& Charbonnel 2005 and these proceedings; Decressin et al. in preparation) by enriching in $\mathrm{O}$ the envelope of intermediate-mass stars already during the second dredge-up event.

We refer to Charbonnel (2005) and to Prantzos \& Charbonnel (2006a, hereafter PC06a) for more details on the caveats of the AGB scenario. What is sure at that point is that the predictive power of the current AGB models is still embedded in considerable difficulties related to the modelling of crucial physical mechanisms (HBB, 3DUP, convection, rotation, mass loss, ...). The most optimistic AGB supporters would certainly claim that the parameter space has not yet been fully explored, and that it is too early to discard the AGB hypothesis. It is clear that the complexity of the physical phenomena at act certainly requires deeper insight. We might be at a stage where the GC data can severely constrain evolutionary AGB models. It could well be also that we have to think about other possible stellar culprits.

\section{The alternative scenario : The winds of rotating massive stars}

The idea that the winds of massive stars (hereafter WMS) may be at the origin of helium anomalies in GC stars has been recently suggested by Norris (2004) and Maeder \& Meynet (2006) in order to explain the blue main sequence of the cluster $\Omega$ Cen: the high helium content of the stars of that sequence could originate from WMS which produce a large helium/metal ratio. The WMS scenario was then proposed by PC06a and further developed by Decressin et al. (2007, hereafter DMCPE07) in the more general context of the GC abundance anomalies described in $\S 1$. 
As shown in Fig. 2, the central temperatures of main sequence massive stars (i.e., with an initial mass higher than $\sim 30 \mathrm{M}_{\odot}$ ) reach the high values required to produce the observed abundance anomalies (see PC06b for more details). In DMCPE06 we computed models of rotating massive stars with the Geneva code, using different sets for the nuclear reaction rates. In the core of main sequence massive stars, the temperature is such that the $\mathrm{CNO}$ cycle is at the equilibrium. There the $\mathrm{C}-\mathrm{N}$ and $\mathrm{O}-\mathrm{Na}$ anticorrelations are easily build, although the extend of the Na production depends on the adopted reaction rates for the $\mathrm{NeNa}$ chain. In addition, $\mathrm{Al}$ is produced. However the rate of the ${ }^{24} \mathrm{Mg}(\mathrm{p}, \gamma)$ reaction has to be increased by a factor of about $10^{3}$ around $50 \times 10^{6} \mathrm{~K}$ with respect to the nominal value published by Iliadis et al. (2001) in order to reproduce the whole amplitude of the observed $\mathrm{Mg}-\mathrm{Al}$ anticorrelation ${ }^{2}$. In that case the central abundances of ${ }^{24} \mathrm{Mg}$ and ${ }^{26} \mathrm{Mg}$ respectively decrease and increase, while ${ }^{25} \mathrm{Mg}$ remains almost constant. These theoretical patterns correspond to those observed presently in GC stars.

In DMCPE06 we suggest that fast rotation on the main sequence allows massive stars to eject the hydrogen-burning products through slow winds which can be easily retained within the GC potential well. As discussed by Meynet in these proceedings, fast rotation velocity has a very important effect on mass loss. Indeed when a star rotates at the break-up velocity (by this, we mean that the equatorial surface velocity is such that the centrifugal acceleration balances the gravity), it loses a very important fraction of its mass through a mechanical wind. This mechanically ejected matter has a very low velocity (of the order of $20 \mathrm{~km} \cdot \mathrm{sec}^{-1}$ or less) and very likely forms an equatorial disk around the star, similar to that observed around Be-type stars.

Fast rotation not only drastically changes (qualitatively and quantitatively) the stellar mass loss. It also modifies, through the meridional circulation and shear turbulence that it induces within the star, the stellar chemical structure as well as the composition at the stellar surface and within the wind. As a consequence the surface abundance variations during the main sequence mimic the central ones described previously, but with some delay. Within the conditions described above (see in particular the assumption on the ${ }^{24} \mathrm{Mg}(\mathrm{p}, \gamma)$ rate), the surface and wind composition of the rotating massive stars present the H-burning signatures observed in GC stars.

These effects are illustrated in Fig. 5 where we show the Kippenhahn diagrams for a $60 \mathrm{M}_{\odot}$ star with $[\mathrm{Fe} / \mathrm{H}]=-1.5$, in both the non-rotating and rotating cases. One clearly sees the impact of rotation on mass loss and on the transport of chemicals from the center up to the surface.

The main reason why massive stars have been discarded in the past is related to the fact that they are expected to produce also heavier elements. Of course within our framework one has to avoid further contamination by the products of He-burning and by the yields in heavy elements of the SNII. Regarding the former ones, DMCPE06 noted that once the star leaves the main sequence its rotation drops. Consequently the wind velocities strongly increases, and the corresponding ejected matter is probably lost by the GC. On the other hand if black holes form in the core collapse they could swallow most of the pre-SN yields (i.e., most of the heavy elements formed after the main sequence phase). More probably, the SN ejection resulting from an initialy fast rotating star, if it occurs, may favor ejection along jets aligned along the rotational axis. Of course these ideas are highly speculative and need to be checked through detailed hydrodynamical simulations.

\footnotetext{
${ }^{2} \mathrm{The}{ }^{24} \mathrm{Mg}(\mathrm{p}, \gamma){ }^{25} \mathrm{Al}$ reaction has a small published rate error in the temperature range between 50 and $80 \mathrm{MK}$ (Powell et al. 1999). However the non-existence of an undetected low-energy resonance can not be proven, although based on the known level structure of ${ }^{25} \mathrm{Al}$ no resonance is predicted.
} 

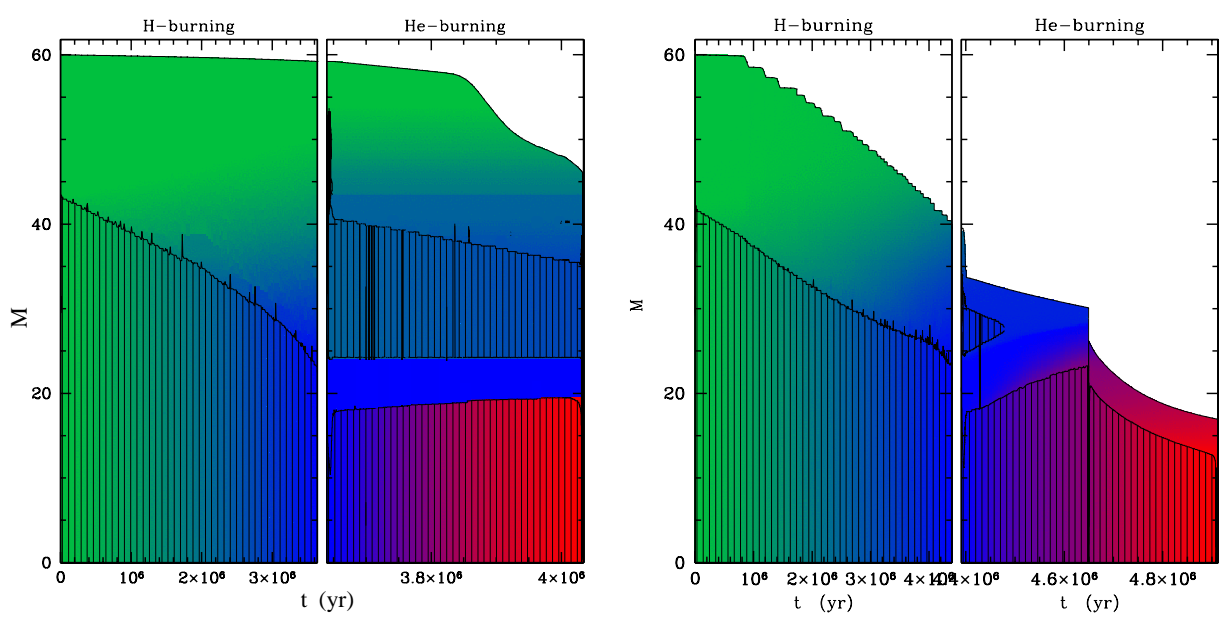

Figure 5: Kippenhahn diagram for a $60 \mathrm{M}_{\odot}$ star with $[\mathrm{Fe} / \mathrm{H}]=-1.5$ in the standard case (Vini = 0; left) and in the fast rotating case $\left(\mathrm{Vini}=800 \mathrm{~km} . \mathrm{sec}^{-1}\right.$; right). The evolution is shown from the zero age main sequence up to the end of central He-burning. The colours indicate the chemical composition ( $\mathrm{H}$ in green, He in blue, $\mathrm{C}$ and $\mathrm{O}$ in red). Courtesy of T.Decressin.

\section{Conclusions}

C-N, O-Na and Mg-Al anticorrelations observed both in turnoff stars and in bright giants in GCs call for a self-enrichment (or pollution) component of the intra-cluster gas. These features are not seen within field stars and appear to be intrinsic properties properties of GCs related to the cluster formation itself. They involve $\mathrm{CNO}$ cycles and $\mathrm{NeNa}$ and $\mathrm{MgAl}$ chains operating at relatively high temperatures.

Today, there is not a unique, neither a well-defined, nor even a fully-consistent scenario for the self-enrichement of GCs. The most commonly invoked one assumes that the H-processed ejecta of massive AGB stars of a first generation form a second stellar generation. This idea suffers from several drawbacks, as discussed in details in PC06a.

On the other hand, the Winds of Fast Rotating Massive Stars scenario presents a very interesting alternative for the understanding of the self-enrichment of GCs. Fast rotation loads the surface layers as well as the stellar wind with the H-burning products. It helps removing that material from the stellar surface through low-velocity stellar winds which can be easily kept within the GC. In addition, massive stars can, through the SN shocks or the ionisation front they produce, trigger star formation in their vicinity. One has then one cause for three effects. Last but not least, it is important to note that the Winds of Fast Rotating Massive Stars scenario for explaining the GC chemical properties is only one consequence, among many others, of the evolution of fast rotating massive stars. For example, that kind of stars might also be at the origin of the carbon rich ultra-metal poor stars or of the high helium abundance in a sub-population of $\Omega$ Cen.

In the framework of the self-enrichment, PC06a presented a new method to derive the Initial Mass Function (IMF) of the polluters, by using the observed O/Na abundance distribution in GCs. We found that the polluter IMF had to be much flatter than presently observed IMFs in stellar 
clusters; this is in agreement with the results of two other methods for GC IMF determination. Additionaly, we find that the present mass of the GC should be totally dominated by stellar remnants if the polluters were AGB stars; this is not the case if the culprits are WMS.

In conclusion, we see that GCs are certainly very useful laboratories to test our understanding of stellar nucleosynthesis, hydrodynamics and evolution in dense environments.

\section{References}

[1] Arnould, M., Goriely, S., Jorissen, A., 1999, A\&A 347, 572

[2] Carretta E., Bragaglia A., Cacciari C., Rossetti E., 2003, A\&A 410, 143

[3] Carretta E., Bragaglia A., Cacciari C., 2004, ApJ 610, L25

[4] Charbonnel, C., 2005, IAU Symposium 228 on "From Lithium to Uranium : Element tracers of early cosmic evolution", Paris, France, Mai 2005, Cambridge University Press, Ed. V.Hill, P.François, F.Primas, p.347

[5] Cohen, J.G., 1978, ApJ, 223, 487

[6] Cottrell P.L., Da Costa G.S., 1981, ApJL, 245, L79

[7] Decressin, T., Charbonnel, C., 2005, IAU Symposium 228 on "From Lithium to Uranium : Element tracers of early cosmic evolution", Paris, France, Mai 2005, Cambridge University Press, Ed. V.Hill, P.François, F.Primas, p.395,

[8] Decressin, T., Meynet, G., Charbonnel, C., Prantzos, N., Ekström, S., 2007, A\&A, in press, astro-ph/0611379 (DMCPE07)

[9] Denissenkov, P.A. \& Denissenkova S.N., 1990, SvA Lett., 16, 275

[10] Denissenkov, P.A. \& Herwig, F., 2003, ApJ, 590, 99

[11] Dickens, R.J., Croke, B.F.W., Cannon, R.D ., Bell, R.A., 1991, Nature, 351, 212

[12] Drake, J.J., Smith, V.V., Suntzeff, N.B., 1992, ApJ 395, L95

[13] Fenner Y., Campbell S., Karakas A.I., Lattanzio J.C., Gibson B.K., 2004, MNRAS, 353, 789

[14] Gratton, R., Sneden, C., Carretta, E., Bragaglia, A., 2000, A\&A, 354, 169

[15] Gratton, R., Bonifacio, P., Bragaglia , A., et al., 2001, A\&A, 369, 87

[16] Grundahl, F., Briley, M., Nissen, P.E., Feltzing, S., 2002, A\&A, 385, L14

[17] Gratton, R., Sneden, C., Carretta, E., 2004, ARAA, 42, 385

[18] Herwig, F., 2004a, ApJ, 605, 425

[19] Herwig, F., 2004b, ApJS, 155, 651 
[20] Iben, I., 1976, ApJ, 208, 165

[21] Iliadis, C., D’Auria, J.M., Starrfield, S., Thompson, W.J., Wiescher, M., 2001, ApJS, 134, 151

[22] Ivans, I.I., Sneden, C., Kraft, R.P., Suntzeff, N.B., Smith, V.V., Langer, G.E., Fulbright, J.P., 1999, AJ, 118, 1273

[23] Karakas, A.I., Lattanzio, J.C., 2003, PASA 20, 279

[24] Langer, G.E., Hoffman, R., Sneden, C., 1993, PASP, 105, 301

[25] Langer, G.E., Hoffman, R., 1995, PASP, 107, 1177

[26] Maeder, A., Meynet, G., 2006, A\&A 448, L37

[27] Mowlavi, N., 1998, AIP Conf. Proc., 425, 507, eds. M.Arnould et al.

[28] Mowlavi, N., Meynet, G., 2000, A\&A 361, 959

[29] Norris, J.E., 2004, ApJ, 612, L25

[30] Norris, J.E., Cottrell, P.L., Freeman, K.C., Da Costa, G.S., 1981, ApJ 244, 205

[31] Osborn, W., 1971, Observatory, 91, 223

[32] Peterson, R.C., 1980, ApJ, 237, L87

[33] Pilachowski, C.A., 1989, in "The abundance spread within globular clusters", eds. G.Cayrel de Strobel, M.Spite, T.L.Evans, p.1

[34] Prantzos, N., Charbonnel, C., 2006a, A\&A in press (PC06a)

[35] Prantzos, N., Charbonnel, C., 2006b, in preparation (PC06b)

[36] Ramirez, S.V. \& Cohen, J.G., 2002, AJ, 123, 3277

[37] Ramirez, S.V. \& Cohen, J.G., 2003, AJ, 125, 224

[38] Shetrone, M.D., 1996, AJ, 112, 1517

[39] Smith, G.H., Shetrone, M.D., Bell, R., Churchill, C.W., Briley, M.M., 1996, AJ, 112, 1511

[40] Smith, V.V., Cunha, K., Ivans, I.I., Lattanzio, J.C., Campbell, S., Hinkle, K.H., 2005, ApJ 633,392

[41] Sneden, C., Gratton, R.G., Crocker, D.A., 1991, A\&A 246, 354

[2005] Sneden, C., 2005, IAU Symp.228 on "From lithium to uranium : Element tracers of early cosmic evolution", Cambridge Univ.Press, Eds.V.Hill, P.François, F.Primas, p.337

[42] Thévenin, F., Charbonnel, C., de Freitas Pacheco, J.A., Idiart, T., Jasniewicz, G., de Laverny, P., Plez, B., 2001, A\&A 373, 905 
[43] Ventura P., D’Antona F., Mazzitelli I., Gratton R., 2001, ApJ 550, L65

[44] Ventura P., D’Antona F., Mazzitelli I., 2002, A\&A 393, 215

[45] Ventura P., D’Antona F., 2005a, A\&A 431, 279

[46] Ventura P., D’Antona F., 2005b, A\&A 439, 1075

[47] Ventura P., D’Antona F., 2005c, ApJ 635, L149

[48] Yong, D., Aoki, W., Lambert, D.L., 2006, ApJ, 638, 1018

[49] Yong, D., Grundahl, F., Lambert, D.L., Nissen, P.E., Shetrone, M.D., 2003, A\&A, 402, 984

[50] Yong, D., Grundahl, F., Nissen, P.E., Jensen, H.R., Lambert, D.L., 2005, A\&A, 438, 875 